\title{
Same Story with Different Endings in HER2- Positive Breast Cancer: Why the Benefit of Pertuzumab is Robust in the Metastatic Scenario and Modest in the Adjuvant Setting?
}

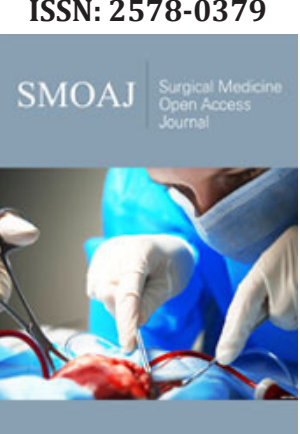

*Corresponding author: Rafael Caparica, Institut Jules Bordet, Université Libre de Bruxelles (U.L.B.), Belgium.

Submission: 沮 December 20, 2019

Published: 漈January 08, 2020

Volume 3 - Issue 2

How to cite this article: Rafael C, Adriana Matutino K, Daniel E, Martine P. Same Story with Different Endings in HER2-Positive Breast Cancer: Why the Benefit of Pertuzumab is Robust in the Metastatic Scenario and Modest in the Adjuvant Setting?. Surg Med Open Acc J.3(2). SMOAJ.000560.2020. DOI: $10.31031 /$ SMOAJ.2020.03.000560.

Copyright@ Rafael Caparica, This article is distributed under the terms of the Creative Commons Attribution 4.0 International License, which permits unrestricted use and redistribution provided that the original author and source are credited.

\author{
Rafael Caparica ${ }^{1 *}$, Adriana Matutino Kahn², Daniel Eiger ${ }^{1}$ and Martine Picca- \\ $\mathrm{rt}^{1}$ \\ ${ }^{1}$ Institut Jules Bordet, Université Libre de Bruxelles (U.L.B.), Belgium. \\ ${ }^{2}$ Department of Internal Medicine, University of Connecticut, USA
}

\begin{abstract}
While the incorporation of pertuzumab to a chemotherapy and trastuzumab backbone (dual HER2 blockade) yielded a robust improvement in the outcomes of HER2-positive metastatic patients in the CLEOPATRA study, in the adjuvant setting the same magnitude of benefit was not reproduced with the addition of pertuzumab in the overall population of the APHINITY study, being the reasons for this discrepancy unknown so far. In the present manuscript, we discuss biological and clinical differences between metastatic and early-stage HER2-positive breast cancer that may potentially explain the different magnitudes of benefit observed with pertuzumab in the different disease settings.
\end{abstract}

Keywords: Breast cancer; HER2; Pertuzumab

\section{Introduction}

The addition of pertuzumab to chemotherapy and trastuzumab yielded an impressive improvement in the outcomes of metastatic HER2-positive breast cancer patients [1]. Intriguingly, the same magnitude of benefit could not be reproduced with pertuzumab in the adjuvant setting, being the reasons for this discrepancy unknown [2,3]. In this manuscript, we discuss clinical and biological differences between metastatic and early-stage HER2-positive breast cancer, and conclude by proposing potential explanations for the distinct magnitudes of benefit of pertuzumab in different disease settings.

\section{Magnitude of Risk Reduction}

When evaluating a new treatment in the context of a clinical trial, events occurring in experimental and control arms are compared [4]. Early-stage HER2-positive breast cancer patients treated with adjuvant chemotherapy and trastuzumab had a $87.8 \%$ recurrencefree survival rate at 6 years as per the recently updated results of the APHINITY trial [3]. In the metastatic setting, however, the perspective is different: only $20 \%$ of patients receiving chemotherapy and trastuzumab remain alive and progression-free at 3 years [1]. Therefore, events are more frequent in the metastatic setting than in early-disease. In other words, there is more room for improvement in metastatic disease, whereas in the adjuvant setting chemotherapy and trastuzumab already yield high Disease-Free Survival (DFS) rates.

Illustrating this hypothesis, the addition of pertuzumab to trastuzumab and chemotherapy in the metastatic setting yields a $32 \%$ relative reduction in the risk of progression, which translates into an 8.2\% absolute increase in Progression-Free Survival (PFS) at 3 years, whereas in the adjuvant setting pertuzumab yields a $24 \%$ relative reduction in the risk of recurrence at 6 years, translating into a modest $2.8 \%$ absolute improvement in invasive DFS (iDFS) [1-3] . When considering only node-positive patients (who present a higher risk of recurrence), the benefit of adjuvant pertuzumab becomes more pronounced ( $28 \%$ relative reduction in recurrence risk yielding a 4.5\% absolute 6-year iDFS improvement) [2,3]. 
In line with this rationale, the KATHERINE study showed improved 3-year iDFS rates with trastuzumab emtansine (TDM1) compared to trastuzumab in HER2-positive breast cancer patients who did not achieve pathologic complete response (pCR) after neoadjuvant treatment $(88.3 \%$ vs. $77.0 \%$; p<0.001) [5]. Importantly, the KATHERINE study enrolled high-risk patients, who are expected to experience more recurrences than the population of the APHINITY study $[2,3,5]$. The different profile of patients enrolled in each study and the fact that T-DM1 may be more active in resistant disease may justify the robust benefit of post-neoadjuvant T-DM1 in the KATHERINE trial and the modest benefit of adjuvant pertuzumab in the overall population of the APHINITY trial $[2,3,5]$.

\section{Gene Expression}

Breast cancer can be divided into four subtypes based on its gene expression profiles, one of which is the HER2-enriched, characterized by a high expression of genes involved in cell proliferation and in HER2 pathway [6]. Concordance between HER2 status assessed by Immunohistochemistry (IHC) or Fluorescent in Situ Hybridization (FISH) and gene expression classification is not perfect: around $65 \%$ of HER2-positive tumors per IHC/FISH are HER2-enriched, whereas $25 \%$ are of the luminal subtypes, and $10 \%$ are basal-like or normal-like. Therefore, HER2-positive disease is clearly a heterogeneous group of tumors $[6,7]$.

HER2-enriched subtype appears to confer increased sensitivity to anti-HER2 treatment, both in early-disease and in metastatic settings $[8,9]$. Interestingly, primary tumors that are not HER2enriched can develop HER2-enriched metastases, suggesting that HER2 expression may change as disease progresses $[10,11]$. Hypothetically, if HER2 expression becomes more frequent as metastases are developed, a more pronounced activity of pertuzumab would be expected in the metastatic setting.

\section{The Immune System}

After binding to HER2, trastuzumab and pertuzumab can induce antibody-mediated cytotoxicity and ultimately promote an anti-tumor immune response [12]. When an antibody binds to its target, its Fragment crystallisable (Fc) region is recognized by $\mathrm{Fc}$ receptors from lymphocytes and antigen-presenting cells, leading to immune activation [13]. Modified anti-HER2 antibodies with impaired Fc domains cannot induce an effective anti-tumor response despite binding adequately to HER2 [14]. In contrast, the activity of anti-HER2 antibodies is enhanced by Fc domains that are more avid for Fc receptors [14]. Single-nucleotide polymorphisms induce structural changes in Fc receptors, which can become more or less avid for the Fc of anti-HER2 antibodies. Therefore, Fc receptor polymorphisms may enhance or compromise the activity of anti-HER2 antibodies.

Studies evaluating Fc receptor polymorphisms as predictive biomarkers for the efficacy of adjuvant trastuzumab presented contradictory results so far $[15,16]$. In the metastatic setting, however, Fc receptor polymorphisms were correlated with increased response to trastuzumab, and also with improved PFS rates in patients treated with the anti-HER 2 antibody margetuximab, suggesting that immune activation may occur in different ways in metastatic and primary HER2-positive breast cancer [17,18]. Given the contradictory results observed in the metastatic and adjuvant settings, Fc polymorphisms are not established as predictive biomarkers in clinical practice.

Tumor Mutation Burden (TMB) represents the amount of mutations per DNA megabase in a specific tumor [19]. High TMB leads to the synthesis of abnormal proteins that can become "neoantigens" recognized by antigen-presenting cells [19]. In breast cancer, TMB is higher in the metastatic setting as compared to early-disease, with HER2-positive and triple-negative subtypes presenting the highest TMB values [20]. Thus, metastatic HER2positive patients have tumors with high TMB, and are probably more prone to benefit from treatments that induce anti-tumor immune responses, such as pertuzumab.

Baseline tumor infiltrating lymphocytes (TILs) levels are correlated with prognosis in HER2-positive breast cancer patients $[21,22]$. However, the role of TILs as predictors of anti-HER2 treatment benefit has yet to be defined: an exploratory study assessed TILs levels in primary tumors and residual disease from 175 HER2-positive patients who received neoadjuvant chemotherapy and trastuzumab: a decrease in TILs levels occurred in $78 \%$ of the patients after neoadjuvant treatment, and was associated with higher pCR rates $(\mathrm{p}<0.001)$. Intriguingly, high TILs levels $(>25 \%)$ in residual disease predicted worse survival $(p=0.009)$ [23]. The reasons why high TILs levels in residual disease may be a bad prognostic factor in HER2-positive disease are unclear, although it could be related to an increase in immunosuppressive cells and a decrease in cytotoxic T cells induced by neoadjuvant treatment [24].

\section{Tumor Heterogeneity}

Tumor cells that harbor a HER2 amplification have an evolutionary advantage in comparison to HER2-negative cells, since HER2-signalling constantly stimulates proliferation and survival [25]. Interestingly, half of circulating tumor cells detected in HER2-positive early-stage breast cancer patients do not express HER2 [26]. Since the blockade of HER2 is the mainstay treatment of HER2-positive breast cancer, the presence of tumor cells that are not dependent on HER2-signalling may lead to treatment resistance [26].

Assuming that most HER2-positive tumors present HER2positive and HER2-negative cells; and disease burden is higher in metastatic patients; as HER2-positive cells have an evolutionary advantage in comparison to HER2-negative cells, a dominance of HER2-positive cells in the advanced disease may occur, particularly in the absence of the selective pressure of systemic therapies. A high proportion of HER2-positive cells may render the tumor more sensitive to HER2 blockade, potentially explaining the more robust benefit of pertuzumab in metastatic disease, especially if we consider the high proportion of de-novo metastatic patients (53.4\%) enrolled in the CLEOPATRA study [1]. Table 1 summarizes potential reasons for the distinct impact of pertuzumab in the adjuvant and metastatic settings. 
Table 1: Potential explanations for the different impact of pertuzumab in the adjuvant and metastatic settings.

\begin{tabular}{|c|c|}
\hline Mechanism & Rationale \\
\hline Magnitudes of Risk Reduction & $\begin{array}{l}\text { Overall in the adjuvant setting, DFS rates are already high with chemotherapy and trastuzumab, thus } \\
\text { there is more margin for improvements in metastatic disease and in high risk early-stage disease } \\
\text { [1-3]. }\end{array}$ \\
\hline Gene Expression & $\begin{array}{l}\text { Metastases from tumours that were not HER2-enriched can present the HER2-enriched profile, which } \\
\text { may be associated with a more pronounced benefit of anti-HER2 treatments }[10,11] \text {. }\end{array}$ \\
\hline Immune Activation & $\begin{array}{l}\text { Metastatic disease may be more immunogenic than } \\
\text { early-disease due to disparities in Fc receptor polymorphisms }[17,18] \text {. }\end{array}$ \\
\hline Tumour Mutational Burden (TMB) & $\begin{array}{l}\text { Metastatic HER2-positive tumours have a high TMB, which may render them more sensitive to treat- } \\
\text { ments that induce an anti-tumour immune response, such as pertuzumab }[23,24] \text {. }\end{array}$ \\
\hline Tumour Heterogeneity & $\begin{array}{l}\text { In treatment-naïve metastatic patients, the dominance of HER2-positive cells over HER2-negative } \\
\text { cells may be more pronounced }[25,26] .\end{array}$ \\
\hline
\end{tabular}

\section{Conclusion}

No irrefutable data can explain the discrepant survival impact yielded by pertuzumab in metastatic and early breast cancer patients, and it is unlikely that one single factor will account for this contrast. Probably tumoral heterogeneity, gene expression patterns at distinct disease stages and differences in TMB account for the heterogeneous benefit of pertuzumab in HER2-positive disease at different stages of evolution. Also, since the induction of an immune response is a mechanism of action of anti-HER2 treatments, the host's immune system and the tumoral microenvironment play also important roles. From a historical perspective, this is not the first time that a significant benefit in the metastatic setting cannot be reproduced in the adjuvant scenario [27]. These findings highlight the importance of evidence-based medicine: A strong biologic rationale and efficacy in the metastatic setting are not a guarantee that the results will be reproducible in early disease. Hence, it is important to have evidence from well-designed randomized trials to support treatment recommendations in each scenario. As the research to depict the molecular characteristics of HER2-positive breast cancer evolves, predictive biomarkers may arise to identify patients who benefit from pertuzumab.

\section{Additional Information}

\section{Ethical approval and consent to participate}

The present work did not perform any experiments with animals or humans; therefore no ethical approval or informed consent forms were necessary.

\section{Consent to publish}

The present work does not contain any patient data in any form; therefore no consent was necessary.

\section{Conflict of Interest}

$\mathrm{RC}$ has received speaking honoraria from Boehringer-Ingelheim and Janssen; and travel grants from Astra-Zeneca and Pfizer. AMK declares no conflicts of interest. DE has received an ESMO Clinical Research Fellowship funded by Novartis. MP is a board member of Radius; has received consultant honoraria from AstraZeneca, Lilly, MSD, Novartis, Odonate, Pfizer, Roche-Genentech, Camel-IDS, Crescendo Biologics, Periphagen, Huya, Debiopharm, PharmaMar, G1 Therapeutics, Menarini, Seattle Genetics, Immunomedics and Oncolytics; RC, DE and MP have received research grants for their Institute from Roche/GNE, Radius, Astra-Zeneca, Lilly, MSD, Novartis, Synthon, Servier, and Pfizer.

\section{Funding}

The present manuscript did not require any funding.

\section{Author's Contribution}

All authors participated in all stages of the present work. The final version of this manuscript was reviewed and approved by all authors before submission.

\section{References}

1. Swain SM, Baselga J, Kim SB, Ro J, Semiglazov V, et al. (2015) Pertuzumab, trastuzumab, and docetaxel in HER2-positive metastatic breast cancer. $\mathrm{N}$ Engl J Med 372(8): 724-734.

2. Minckwitz G, Procter M, Azambuja E, Zardavas D, Benyunes M, et al. (2017) Adjuvant pertuzumab and trastuzumab in early HER2-positive breast cancer. N Engl J Med 377(2): 122-131.

3. Piccart M (2019) Interim overall survival analysis of Aphinity (BIG 4-11): A randomized multicenter, double-blind, placebo-controlled trial comparing chemotherapy plus trastuzumab plus pertuzumab versus chemotherapy plus trastuzumab plus placebo as adjuvant therapy in patients with operable HER2-positive early breast cancer. USA. 
4. Umscheid CA, Margolis DJ, Grossman CE (2011) Key concepts of clinical trials: A narrative review. Postgrad Med 123(5): 194-204.

5. Minckwitz G, Huang CS, Mano MS, Loibl S, Mamounas EP, et al. (2018) Trastuzumab emtansine for residual invasive HER2-positive breast cancer. N Engl J Med 380(7): 617-628.

6. Perou CM, Sørlie T, Eisen MB, Jeffrey SS, Rees CA, et al. (2000) Molecular portraits of human breast tumors. Nature 406(6797): 747-752.

7. Prat A, Carey LA, Adamo B, Vidal M, Tabernero J, et al. (2014) Features and survival outcomes of the intrinsic subtypes within HER2-positive breast cancer. JNCI J Natl Cancer Inst 106(8).

8. Llombart CA, Cortés J, Paré L, Galván P, Bermejo B, et al. (2017) HER2enriched subtype as a predictor of pathological complete response following trastuzumab and lapatinib without chemotherapy in earlystage HER2-positive breast cancer (PAMELA): An open-label, singlegroup, multicenter, phase 2 trial. Lancet Oncol 18(4): 545-554.

9. Prat A, Pascual T, Angelis C, Wang T, Cortés J, et al. (2019) HER2-enriched subtype and ERBB2 expression in HER2-positive breast cancer treated with dual HER2 blockade. J Natl Cancer Inst.

10. Stefanovic S, Wirtz R, Deutsch TM, Hartkopf A, Sinn P, et al. (2017) Tumor biomarker conversion between primary and metastatic breast cancer: mRNA assessment and its concordance with immunohistochemistry. Oncotarget 8(31): 51416-51428.

11. Priedigkeit N, Hartmaier RJ, Chen Y, Vareslija D, Basudan A, et al. (2017) Intrinsic subtype switching and acquired ERBB2 / HER2 amplifications and mutations in breast cancer brain metastases. JAMA Oncol 3(5): 666671.

12. Collins DM, Donovan N, Gowan PM, Sullivan F, Duffy MJ, et al. (2012) Trastuzumab induces Antibody-Dependent Cell-mediated Cytotoxicity (ADCC) in HER-2-non-amplified breast cancer cell lines. Ann Oncol 23(7): 1788-1795.

13. Vogelpoel LTC, Baeten DLP, Jong EC, Dunnen J (2015) Control of cytokine production by human Fc gamma receptors: Implications for pathogen defense and autoimmunity. Front Immunol 6: 79.

14. Clynes RA, Towers TL, Presta LG, Ravetch JV (2000) Inhibitory Fc receptors modulate in-vivo cytoxicity against tumortargets. Nat Med 6(4): 443-446.

15. Norton N, Olson RM, Pegram M, Tenner K, Ballman KV (2014) Association studies of Fc receptor polymorphisms with outcome in HER2+ breast cancer patients treated with trastuzumab in NCCTG (Alliance) trial N9831. Cancer Immunol Res 2(10): 962-969.

16. Gavin PG, Song N, Kim SR, Lipchik C, Johnson NL, et al. (2017) Association of polymorphisms in FCGR2A and FCGR3A with degree of trastuzumab benefit in the adjuvant treatment of ERBB2/HER2-Positive breast cancer: Analysis of the NSABP B-31 trial. JAMA Oncol 3(3): 335-341.
17. Hope S, Mark DP, William JG, Javier C, Giuseppe C, et al. (2016) SOPHIA: Phase 3, randomized study of Margetuximab (M) plus Chemotherapy (CTX) vs Trastuzumab (T) plus CTX in the treatment of patients with HER2+ Metastatic Breast Cancer (MBC). J Clin Oncol 34(15_suppl): TPS630.

18. Shimizu C, Mogushi K, Morioka MS, Yamamoto H, Tamura K, et al. (2016) Fc-Gamma receptor polymorphism and gene expression of peripheral blood mononuclear cells in patients with HER2-positive metastatic breast cancer receiving single-agent trastuzumab. Breast Cancer 23(4): 624-632.

19. Chalmers ZR, Connelly CF, Fabrizio D, Gay L, Ali SM (2017) Analysis of 100,000 human cancer genomes reveals the landscape of tumor mutational burden. Genome Med 9(1): 34 .

20. Barroso SR, Jain E, Kim D, Partridge AH, Cohen O, et al. (2018) Determinants of high Tumor Mutational Burden (TMB) and mutational signatures in breast cancer. J Clin Oncol 36(15_suppl): 1010.

21. Denkert C, Minckwitz G, Darb ES, Lederer B, Heppner BI, et al. (2018) Tumour-infiltrating lymphocytes and prognosis in different subtypes of breast cancer: A pooled analysis of 3771 patients treated with neoadjuvant therapy. Lancet Oncol 19(1): 40-50.

22. Luen SJ, Salgado R, Fox S, Savas P, Wong EJ, et al. (2017) Tumourinfiltrating lymphocytes in advanced HER2-positive breast cancer treated with pertuzumab or placebo in addition to trastuzumab and docetaxel: A retrospective analysis of the cleopatra study. Lancet Oncol 18(1): 52-62.

23. Hamy AS, Pierga JY, Sabaila A, Cottu P, Lerebours F, et al. (2017) Stromal lymphocyte infiltration after neoadjuvant chemotherapy is associated with aggressive residual disease and lower disease-free survival in HER2-positive breast cancer. Ann Oncol 28(9): 2233-2240.

24. Luen SJ, Griguolo G, Nuciforo P, Christine C, Roberta F, et al. (2019) Ontreatment changes in Tumor-Infiltrating Lymphocytes (TIL) during neoadjuvant HER2 therapy (NAT) and clinical outcome. J Clin Oncol 37(15_suppl): 574-574.

25. Yarden Y (2001) Biology of HER2 and its importance in breast cancer. Oncology 61(Suppl 2): 1-13.

26. Jaeger BAS, Neugebauer J, Andergassen U (2017) The HER2 phenotype of circulating tumor cells in HER2-positive early breast cancer: A translational research project of a prospective randomized phase III trial. PLOS ONE 12(6): e0173593.

27. Kümler I, Christiansen OG, Nielsen DL (2014) A systematic review of bevacizumab efficacy in breast cancer. Cancer Treat Rev 40(8): 960-973.

For possible submissions Click below: 